\title{
Human Papillomavirus Infection in Female Sex Workers: A Case Control Study
}

\author{
Nadia Velazquez-Hernandez ${ }^{a}$, Luis Francisco Sanchez-Anguiano ${ }^{a}$, Fernando Martin Guerra-Infante ${ }^{\mathrm{b}}$, \\ Marisela Aguilar-Duran ${ }^{\mathrm{a}}$, Alma Rosa Perez-Alamos ${ }^{\mathrm{a}}$, Sergio Estrada-Martinez ${ }^{\mathrm{a}}$, \\ Jose Antonio Navarrete-Flores ${ }^{\mathrm{a}}$, Ada Agustina Sandoval-Carrillo ${ }^{\mathrm{a}}$, \\ Elizabeth Irasema Antuna-Salcido a, Jesus Hernandez-Tinococ, \\ Cosme Alvarado-Esquivel ${ }^{c, d}$
}

\begin{abstract}
Background: To determine the association of infection with human papillomavirus (HPV) and the occupation of female sex worker; and the correlation of infection with HPV with sociodemographic, clinical and behavioral characteristics of female sex workers.

Methods: We performed a case-control study of 217 female sex workers and 354 women without sex work in Durango City, Mexico. We determined the prevalence of infection with HPV in cervical samples of women using polymerase chain reaction, and HPV genotypes were determined using line probe assay. Bivariate and multivariate analyses were used to assess the association between the characteristics of women and infection.

Results: Twelve (5.5\%) of the 217 sex workers, and 10 (2.8\%) of the 354 control women were positive for HPV DNA (age-adjusted OR = 1.51; $95 \%$ CI: 0.62 - 3.68; P=0.36). Six $(50.0 \%)$ of the 12 HPV DNA positive sex workers had infections with high-risk genotypes $(16,31$, $33,35,51,58)$. Seven $(70 \%)$ of the $10 \mathrm{HPV}$ DNA positive control women had infections with high-risk genotypes $(16,18,56,58$, and 66). The frequency of high risk genotypes in the control women was equal with that found in the female sex workers $(P=0.41)$. Logistic regression analysis showed that the variable alcohol consumption was associated with HPV infection ( $\mathrm{OR}=4.0 ; 95 \% \mathrm{CI}$ : 1.0 - 16.0; $\mathrm{P}=0.04)$.
\end{abstract}

Conclusions: No association between HPV infection and female sex

Manuscript submitted December 30, 2018, accepted January 25, 2019

anstitute for Scientific Research "Dr. Roberto Rivera-Damm", Juarez University of Durango State, Avenida Universidad S/N, 34000 Durango, Mexico bDepartamento de Infectologia, Laboratorio de Virologia del Instituto Nacional de Perinatologia, Departamento de Microbiologia de la Escuela Nacional de Ciencias Biologicas, IPN, D.F., Mexico

'Faculty of Medicine and Nutrition, Juarez University of Durango State, Avenida Universidad S/N, 34000 Durango, Mexico

${ }^{\mathrm{d} C}$ Corresponding Author: Cosme Alvarado-Esquivel, Biomedical Research Laboratory, Faculty of Medicine and Nutrition, Juarez University of Durango State, Avenida Universidad S/N, 34000 Durango, Dgo, Mexico.

Email: alvaradocosme@yahoo.com

doi: https://doi.org/10.14740/jocmr3739 work was found in our setting. High risk HPV genotypes were prevalent among the women studied. Results can be used for the design of preventive measures against HPV infection.

Keywords: Human papillomavirus; Prevalence; Female sex workers; Case-control study

\section{Introduction}

Human papillomavirus (HPV) is a double-stranded DNA virus causing infections in humans [1]. Epithelial cells of the skin and mucosa are targets for infection with this virus [2]. Infection with HPV may lead to cervical and oropharyngeal cancers [3]. Cervical cancer is the third most common malignancy in women and persistent infection with HPV is the major risk factor for the development of this disease [4]. There are over 200 HPV genotypes, but only a subset of HPV variants so called "high-risk" HPV genotypes cause cervical disease [5]. Fourteen HPV genotypes are considered high risk types including HPV 16, 18, 31, 33, 35, 39, 45, 51, 52, 56, 58, 59,66 , and $68[6,7]$. HPV 16 and 18 genotypes are the most common types [7]. HPV is transmitted by direct contact [8], usually by sexual intercourse [9]. HPV causes the most common sexually transmitted infection [10]. Female sex workers have a high prevalence of infection with high risk HPV genotypes [11]. These workers constitute an epidemiologically important population group for HPV infection because they may acquire this infection and transmit it to other sexual partners.

The epidemiology of HPV infection in female sex workers in Mexico has been poorly studied. In a previous study of female sex workers in the northern Mexican city of GomezPalacio in Durango State, researchers found a $28.57 \%$ prevalence of HPV infection in 168 sex workers studied using polymerase chain reaction (PCR) [12]. In the present study, that was performed in another city (the capital) of Durango State, and with a different study design (case-control) from the previous study (cross-sectional), we aimed to determine: 1) The association between the occupation of female sex work and 
infection with HPV in the northern Mexican city of Durango; 2) The HPV genotypes infecting the women studied; and 3) The HPV prevalence association with behavioral factors of the female sex workers studied.

\section{Materials and Methods}

\section{Study design}

We performed a case-control study. Cases were female sex workers, and controls were women without the occupation of sex worker. Controls were matched with cases by sex (females). This study was performed from November 2014 to May 2016.

\section{Description of the population groups studied}

Cases included female sex workers registered at the Municipal Clinic for Sanitary Inspection in Durango City, Mexico. All registered female sex workers were invited to participate. A female sex worker was defined as a woman working at the sex industry. Inclusion criteria for enrollment of cases were: 1) Registered female sex worker; 2) With at least 1 year of working in the sex activity; and 3) Aged 18 years and older.

Controls included sexually active women but without sex work attended at the Clinic for Family Planning in the Institute for Scientific Research of the Juarez University of Durango State in Durango City, Mexico.

\section{Sociodemographic, obstetric and behavioral data of female sex workers}

A questionnaire was used to record the sociodemographic, obstetric and behavioral data of female sex workers. Items about age, education, and obstetric data as pregnancies, deliveries, cesarean sections, and miscarriages were included in the questionnaire. In addition, behavioral factors as duration in the activity (years), condom use, contact with semen during vaginal intercourse, practice of oral or anal sex, area of work, sex activity in Mexican States other than Durango or abroad, injuries during sex activity, alcohol or tobacco consumption, and drug use from the female sex workers were obtained.

\section{Laboratory tests}

A cervical swap sample was obtained from each participant. Samples were kept in 2SP medium and stored at $-70{ }^{\circ} \mathrm{C}$ until analysis. DNA of samples was extracted by proteinase $\mathrm{K}$ digestion, and phenol-chloroform extraction. Detection of HPV DNA was performed by PCR using the primers MY09/11. The amplification mixture contained $2 \mathrm{mM} \mathrm{MgCl}_{2}, 0.2 \mu \mathrm{M}$ of primers, 2 units of Taq polymerase, $0.2 \mathrm{mM}$ of dNTPs, $2 \mathrm{ng}$ of DNA, and buffer in a $50 \mu \mathrm{L}$ reaction volume. Amplifications were performed for 39 cycles with the following cycling pa- rameters: $94{ }^{\circ} \mathrm{C}$ for $1 \mathrm{~min}, 55^{\circ} \mathrm{C}$ for $2 \mathrm{~min}$, and $72{ }^{\circ} \mathrm{C}$ for 2.5 min. PCR products were run in $2 \%$ agarose gels and stained with ethidium bromide.

Genotyping of HPV DNA was performed by a commercially available line probe assay kit: INNO-LiPA HPV Genotyping Extra (INNOGENETICS N.V. Gent, Belgium). This test is based on the principle of reverse hybridization and allows the detection of 28 different HPV genotypes. This genotyping assay uses biotinylated primers (SPF10) to amplify the L1 region of HPV. Amplicons are denatured and hybridized with specific oligonucleotide probes on membrane strips. Streptavidin-conjugated alkaline phosphatase was added and incubation with BCIP/NBT chromogen yielded a purple precipitate. Results were interpreted visually. All assays were performed following the instructions of the manufacturer.

\section{Statistical analysis}

The statistical analysis was performed with the software SPSS for Windows version 15.0 (SPSS Inc. Chicago, Illinois) and Epi Info 7. Calculation of the sample size was performed using the following data: $95 \%$ two-sided confidence level, a power of $80 \%$, a $1: 1.5$ ratio of cases and controls, a reference seroprevalence of $16.67 \%$ [13] as the percentage outcome in unexposed group, and an odds ratio (OR) of 2.0. The result of the sample size calculation was 172 cases and 258 controls. Descriptive statistics were used to calculate the prevalence of HPV infection. The risk of HPV infection was calculated by the OR with $95 \%$ confidence interval (CI) adjusted by age. The association between HPV infection and the characteristics of female sex workers was assessed in available data by the Pearson's Chi-squared test and the Fisher exact test (when values were small). Characteristics of female sex workers with a $\mathrm{P}$ value $\leq 0.20$ obtained by bivariate analysis were selected for multivariate analysis. ORs and 95\% CIs were calculated by logistic regression with the Enter method. A P value $<0.05$ was considered statistically significant.

\section{Ethics approval}

This project was approved by the Ethics Committee of the Institute for Scientific Research of Juarez University of Durango State, Mexico. Participation in this study was voluntary, and a written informed consent was obtained from all participants.

\section{Results}

A total of 571 women were included in the study. Of them, 217 were sex workers and 354 were control subjects. Sex workers were $32.4 \pm 9.9$ (range: $18-67$ ) years old. Whereas control women were $36.4 \pm 10.0$ (range: 18 - 67) years old. Control women were significantly older than sex workers $(\mathrm{P}<0.01)$. Therefore, statistical analysis was performed with age adjustment. Of the 217 sex workers, 12 (5.5\%) were positive for HPV 
Table 1. Correlation of HPV Infection and General Sociodemographic and Obstetric Characteristics of Female Sex Workers and Controls

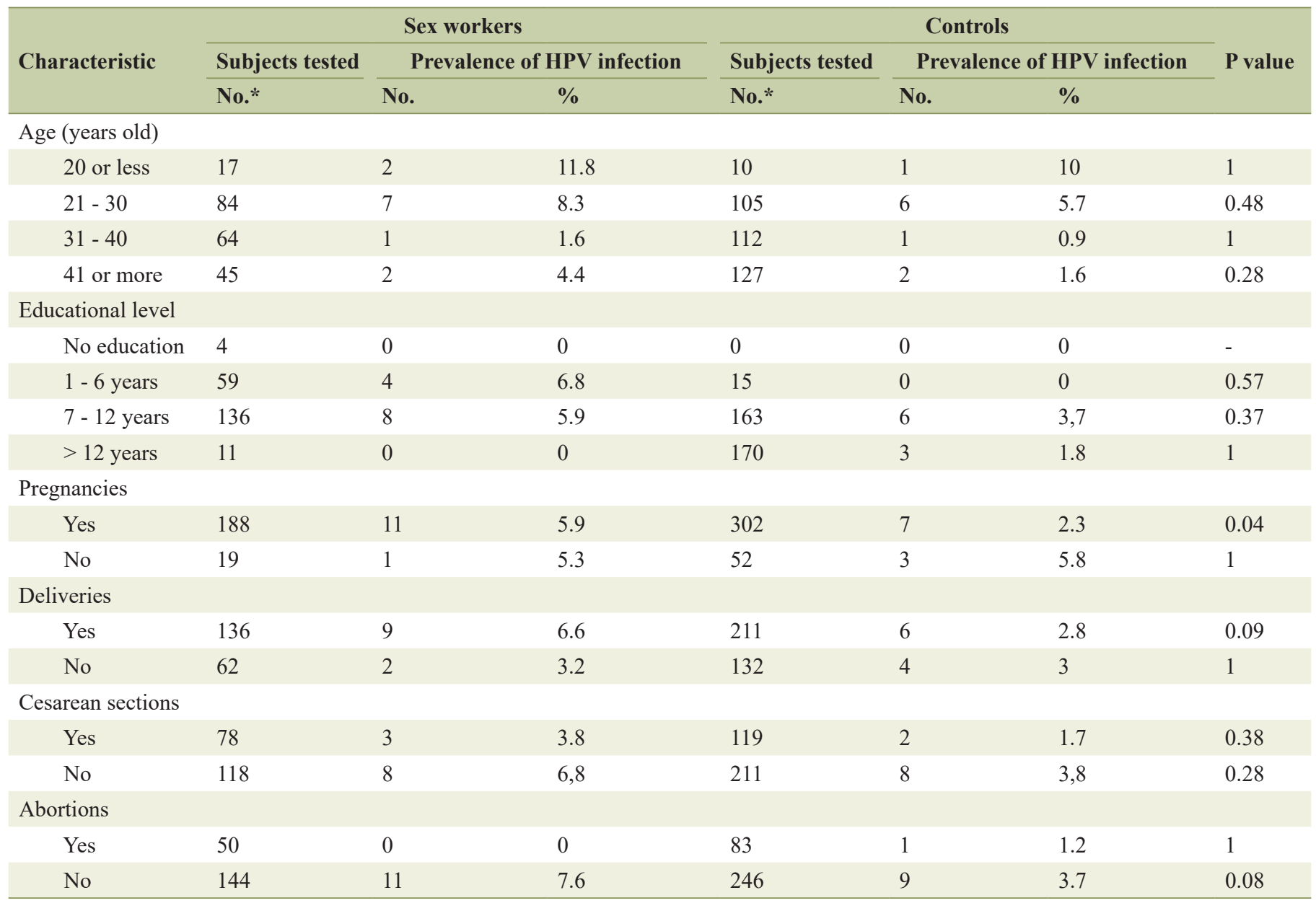

*Sums may not add up to 217 cases or 354 controls because of some missing values.

DNA detection by PCR. Whereas of the 354 control women, $10(2.8 \%)$ were positive for PCR. No statistically significant difference in the frequencies of HPV DNA detection between sex workers and control subjects was found (age-adjusted OR $=1.65 ; 95 \% \mathrm{CI}: 0.69-3.96 ; \mathrm{P}=0.25)$.

Of the 12 HPV DNA positive female sex workers, eight had single infections and four double or triple infections. HPV genotypes found in the eight sex workers with single infections were: $6,11,16,26,35,40,43$, and 51. HPV genotypes found in the four sex workers with multiple infections were: 69 and 71 in two; 31, 40, and 58 in one; and 31, 33, and 53 in one. Six $(50.0 \%)$ of the 12 HPV DNA positive sex workers had infections with high-risk genotypes $(16,31,33,35$, $51,58)$; whereas of the $10 \mathrm{HPV}$ positive control women, eight had infection with a single HPV genotype, and two had infections with two or three HPV genotypes. Single HPV genotypes found in eight HPV DNA positive control women were: genotype 16 in two, genotype 18 in two, genotype 66 in one, and genotype 40 in three. HPV genotypes found in the two control women with double or triple infections were genotypes 56 and 58 in one, and genotypes 31,40 , and 58 in the other one. Seven
$(70 \%)$ of these 10 control women had infections with high-risk genotypes $(16,18,56,58$, and 66). The frequency of high-risk genotypes in the control women was equal to that found in the female sex workers $(\mathrm{P}=0.41)$.

Stratification of socioeconomic and obstetric characteristics including age, educational level, and history of pregnancies, deliveries, cesarean sections, and miscarriages showed a similar $(\mathrm{P}>0.05)$ rate of HPV positivity in female sex workers and controls (Table 1). None of these characteristics was associated with HPV infection. Of the behavioral data (Table 2), the variables time of sexual activity ( $\leq 5$ years), sex activity in Mexican States other than Durango and alcohol consumption had $\mathrm{P}$ values $\leq 0.20$ by bivariate analysis and were selected for multivariate analysis. Other behavioral characteristics including condom use, contact with semen during vaginal intercourse, practice of oral or anal sex, area of work, sex activity abroad, injuries during sex activity, tobacco consumption, and drug use had $\mathrm{P}$ values $>0.20$ by bivariate analysis. Results of the logistic regression analysis are shown in Table 3. Only the variable alcohol consumption was associated with HPV infection $(\mathrm{OR}=4.0 ; 95 \% \mathrm{CI}: 1.0-16.0 ; \mathrm{P}=0.04)$. 
Table 2. Results of Bivariate Analysis of Behavioral Characteristics of Female Sex Workers and Infection With HPV

\begin{tabular}{lllll}
\hline \multirow{2}{*}{ Characteristic } & \multicolumn{2}{c}{ Subjects tested } & & \multicolumn{2}{c}{ Prevalence of HPV infection } \\
\cline { 2 - 4 } & No.* & No. & $\%$ value
\end{tabular}

Time of sexual activity (years)

$\begin{array}{lllll}1 \text { to } 5 & 25 & 3 & 12 & 0.07 \\ 6 \text { to } 11 & 51 & 5 & 9.8 & 3 \\ 11 \text { or more } & 134 & 4 & & 0.5 \\ \text { ndom use } & & & 6.7 & 0.54 \\ \text { Yes } & 119 & 8 & 4.7 & \end{array}$

Contact with semen during vaginal intercourse

$\begin{array}{llll}\text { Yes } & 90 & 5 & 5.6 \\ \text { No } & 118 & 7 & 5.9\end{array}$

Practice of oral sex

$\begin{array}{llllll}\text { Yes } & 126 & 5 & 4 & 0.22 \\ \text { No } & 83 & 7 & 8.4 & \end{array}$

Practice of anal sex

$\begin{array}{lllll}\text { Yes } & 18 & 0 & 0 & 0.6 \\ \text { No } & 190 & 12 & 6.3 & \end{array}$

Area of work

$\begin{array}{lllll}\text { Urban } & 205 & 12 & 5.9 & 0.91 \\ \text { Suburban } & 2 & 0 & 0 & 0 \\ \text { Rural } & 1 & 0 & 0\end{array}$

Sex activity in Mexican states other than Durango

$\begin{array}{lllll}\text { Yes } & 59 & 1 & 1.7 & 0.18 \\ \text { No } & 150 & 11 & 7.3 & \end{array}$

Sex activity abroad

$\begin{array}{llll}\text { Yes } & 4 & 0 & 0 \\ \text { No } & 206 & 12 & 5.8\end{array}$

Injuries during sex activity

$\begin{array}{ccccc}\text { Yes } & 13 & 0 & 0 & 1 \\ \text { No } & 195 & 12 & 6.2\end{array}$

Alcohol consumption

$\begin{array}{lllll}\text { Yes } & 36 & 4 & 11.1 & 0.11 \\ \text { No } & 181 & 8 & 4.4 & \end{array}$

Tobacco consumption

\begin{tabular}{ccccc} 
Yes & 42 & 1 & 2.4 & 0.46 \\
No & 175 & 11 & 6.3 & \\
Drug use & & & & \\
Yes & 13 & 1 & 7.7 & 0.53 \\
No & 204 & 11 & 5.4 & \\
\hline
\end{tabular}

*Sums may not add up to 217 because of some missing values. 
Table 3. Results of the Multivariate Analysis of a Selection of Characteristics of Female Sex Workers and Infection With HPV.

\begin{tabular}{llll} 
Characteristic & Odds ratio & $\mathbf{9 5 \%}$ Confidence interval & P value \\
\hline Alcohol consumption & 4 & $1.0-16.0$ & 0.04 \\
Time of sexual activity ( $\leq 5$ years) & 3.4 & $0.79-15.3$ & 0.09 \\
Sex activity in Mexican states other than Durango & 0.18 & $0.02-1.5$ & 0.11 \\
\hline
\end{tabular}

\section{Discussion}

There is scanty information about the epidemiology of HPV infection in female sex workers in Mexico. Therefore, we sought to determine the association of HPV infection and female sex work in Durango City, Mexico. Comparison of the prevalence of HPV infection obtained by PCR indicates that female sex workers had a similar prevalence of HPV infection to control women. This result thus suggests that female sex workers do not have an increased risk of HPV infection. This finding is unexpected since having multiple sexual partners may lead to higher HPV transmission, and in a review of 35 studies, researchers found that female sex workers had a high prevalence of HPV infection [11]. In a study in Antwerp, Belgium, researchers found a higher prevalence of high-risk HPV infection in female sex workers than in controls of the general population [14]. Similarly, in a study in Hungary, higher prevalences of HPV infection and high-risk HPV infection were found in female sex workers than in control women [15]. Furthermore, in a study in Japan, researchers found a higher prevalence of intermediate- and high-risk HPV infection in commercial sex workers than in control subjects [16]. On the other hand, the lack of association between HPV infection and sex work found in our study agrees with a similar result found in a study of commercial sex workers in Australia [17]. In a national context, we are aware of only two previous reports on the epidemiology of HPV infection in female sex workers in Mexico. In a previous descriptive study of female sex workers in the northern Mexican city of Gomez-Palacio in Durango State, researchers found a high $(28.57 \%)$ frequency of HPV infection in 168 sex workers who have practiced prostitution for more than 5 years [12]. In a study of female sex workers from Mexico City, high prevalences of HPV infection (48.9\%), and of high-risk HPV genotypes (43\%) were found [18]. The prevalence of HPV infection found in our study is thus much lower than those reported in previous studies in female sex workers in Mexico. It is not clear why female sex workers in Durango City had lower prevalence of HPV infection than those in Gomez-Palacio City and Mexico City. It is likely that differences in the sociodemographic or behavioral characteristics of sex workers including age, duration in the activity, condom use, or number of sexual partners might explain the differences in prevalences among the studies. On the other hand, we compared the prevalence of HPV infection found in sex workers from Durango City to those reported in women in the region. The low prevalence of HPV infection in sex workers found in our study is comparable to the $4.8 \%$ prevalence reported in women seeking for cervical Papanicolaou examination in three public health centers in Durango City [19], and the $2.15 \%$ prevalence found in women with normal cyto-histopathologic diagnosis in Gomez-Palacio City [13].

The frequency of high-risk HPV genotypes found in our study $(50 \%)$ is higher than the $11.90 \%$ prevalence of high-risk HPV genotypes reported in female sex workers in GomezPalacio City, Durango [12], and comparable to the $43 \%$ prevalence reported in sex workers from Mexico City [18].

We found that the variable alcohol consumption was associated with HPV infection in female sex workers. To the best of our knowledge, there is no previous report on the association between female sex work and alcohol consumption. It is possible that sex workers with alcohol consumption take less care of preventive measures against HPV infection. Substance use including alcohol consumption has been linked to HPV infection in male sex workers in Vietnam [20].

\section{Conclusions}

We conclude that HPV infection is not associated with female sex work in Durango City. Female sex workers in Durango City have a low prevalence of HPV infection but a high prevalence of high-risk HPV genotypes. The association between HPV infection and alcohol consumption deserves further investigation.

\section{Financial Support}

This work was financially supported by Juarez University of Durango State, Mexico.

\section{Conflict of Interest}

No competing interests exist.

\section{References}

1. de Sanjose S, Brotons M, Pavon MA. The natural history of human papillomavirus infection. Best Pract Res Clin Obstet Gynaecol. 2018;47:2-13.

2. Prigge ES, von Knebel Doeberitz M, Reuschenbach M. Clinical relevance and implications of HPV-induced neoplasia in different anatomical locations. Mutat Res Rev Mutat Res. 2017;772:51-66.

3. Berman TA, Schiller JT. Human papillomavirus in cervical cancer and oropharyngeal cancer: One cause, two diseases. Cancer. 2017;123(12):2219-2229.

4. Bahrami A, Hasanzadeh M, Shahidsales S, Farazestanian 
M, Hassanian SM, Moetamani Ahmadi M, Maftouh M, et al. Genetic susceptibility in cervical cancer: From bench to bedside. J Cell Physiol. 2018;233(3):1929-1939.

5. Graham SV. The human papillomavirus replication cycle, and its links to cancer progression: a comprehensive review. Clin Sci (Lond). 2017;131(17):2201-2221.

6. Egawa N, Egawa K, Griffin H, Doorbar J. Human Papillomaviruses; Epithelial Tropisms, and the Development of Neoplasia. Viruses. 2015;7(7):3863-3890.

7. Choi YJ, Park JS. Clinical significance of human papillomavirus genotyping. J Gynecol Oncol. 2016;27(2):e21.

8. Schiffman M, Doorbar J, Wentzensen N, de Sanjose S, Fakhry C, Monk BJ, Stanley MA, et al. Carcinogenic human papillomavirus infection. Nat Rev Dis Primers. 2016;2:16086.

9. Carter JR, Ding Z, Rose BR. HPV infection and cervical disease: a review. Aust N Z J Obstet Gynaecol. 2011;51(2):103-108.

10. Sehnal B, Rozsypal H, Nipcova M, Slama J. [The prevalence, incidence, persistence and transmission ways of human papillomavirus infection (HPV)]. Epidemiol Mikrobiol Imunol. 2017;66(4):198-209.

11. Soohoo M, Blas M, Byraiah G, Carcamo C, Brown B. Cervical HPV Infection in Female Sex Workers: A Global Perspective. Open AIDS J. 2013;7:58-66.

12. Rodriguez-Reyes ER, Quinonez-Perez JM, Cerda-Flores RM, Saucedo-Cardenas O, Cortes-Gutierrez EI. [Prevalence of HPV in sex workers in Durango, Mexico]. Salud Publica Mex. 2005;47(6):393.

13. Rodriguez-Reyes ER, Cerda-Flores RM, Solis Rios NP, Quinones Perez JM, Cortes-Gutierrez EI. [Identification and typification of the human papilloma virus in women using the "Timely Detection of Cancer" program in Du- rango, Mexico]. Ginecol Obstet Mex. 2003;71:471-475.

14. Vorsters A, Cornelissen T, Leuridan E, Bogers J, Vanden Broeck D, Benoy I, Goossens H, et al. Prevalence of highrisk human papillomavirus and abnormal pap smears in female sex workers compared to the general population in Antwerp, Belgium. BMC Public Health. 2016;16:477.

15. Marek E, Dergez T, D'Cruz G, Bozsa S, Cseh A, Szilard I, Benczik M, et al. Human papillomavirus infections among Hungarian female sex workers. Eur J Cancer Care (Engl). 2014;23(1):65-75.

16. Ishi K, Suzuki F, Saito A, Kubota T. Prevalence of human papillomavirus, Chlamydia trachomatis, and Neisseria gonorrhoeae in commercial sex workers in Japan. Infect Dis Obstet Gynecol. 2000;8(5-6):235-239.

17. Tideman RL, Thompson C, Rose B, Gilmour S, Marks C, van Beek I, Berry G, et al. Cervical human papillomavirus infections in commercial sex workers-risk factors and behaviours. Int J STD AIDS. 2003;14(12):840-847.

18. Juarez-Figueroa LA, Wheeler CM, Uribe-Salas FJ, Conde-Glez CJ, Zampilpa-Mejia LG, Garcia-Cisneros S, Hernandez-Avila M. Human papillomavirus: a highly prevalent sexually transmitted disease agent among female sex workers from Mexico City. Sex Transm Dis. 2001;28(3):125-130.

19. Sanchez-Anguiano LF, Alvarado-Esquivel C, ReyesRomero MA, Carrera-Rodriguez M. Human papillomavirus infections in women seeking cervical Papanicolaou cytology of Durango, Mexico: prevalence and genotypes. BMC Infect Dis. 2006;6:27.

20. Yu G, Clatts MC, Goldsamt LA, Giang le M. Substance use among male sex workers in Vietnam: prevalence, onset, and interactions with sexual risk. Int J Drug Policy. 2015;26(5):516-521. 